\title{
ONE-DIMENSIONAL EQUATIONS AND THEIR SOLUTIONS MODELLING A HOMOGENEOUSLY BROADENED, FREQUENCY CONTINUUM, INJECTION MASER*
}

\author{
BY \\ I. LERCHE \\ University of South Carolina
}

\begin{abstract}
The strongly coupled, nonlinear, differential equations describing the amplification of intensity for propagation of a broad-band signal through a homogeneously broadened amplifier are reduced to a simple linear integral equation which is solved by conventional Laplace transform techniques.

The frequency-dependent intensity is shown to be generally expressible in terms of simple quadratures taken over the solution to the linear integral equation. We have done these calculations to aid those interested in astrophysical masers, such as $O H$ sources, in particular; and as a guide to a technique which may be of greater validity than for just the simple one-dimensional situation recorded here.
\end{abstract}

I. Introduction. Recently, Cassidy (1984) has noted that "To describe fully the spectral properties on an injection laser amplifier (Marcuse, 1983) or injection laser (Lau and Yariv, 1982) the nonuniform distribution of the photon and the carrier densities along the length of the device must be taken into account." In addition Cassidy provided an exact analytic solution (in parametric form) to the one-dimensional steady-state laser equations in the limit $n_{1}=0$, i.e., when the lower-level population is pumped rapidly. Motivated by Cassidy's work, the general solution to the one-dimensional steady-state laser equations has recently been found (Lerche, 1985). The difficulty in applying these recent results to laser problems is that the calculations deal with a denumerable sum of modes while it is often the case (see, e.g., Cook, 1979; Goldreich and Keeley, 1972) that a continuum distribution of frequencies is considered to represent better observed laser and maser activity. This is particularly so in astrophysical situations.

It would then seem appropriate to ask whether the laser equations are solvable under the more general conditions of a continuum frequency distribution of modes. And it is also of interest to compare and contrast the formal behavior when a continuum of modes is under consideration against the results for the denumerable sum of modes.

\footnotetext{
${ }^{*}$ Received September 17, 1984.
} 
The purpose of this paper is to demonstrate that the laser equations for a continuum distribution of modes are indeed solvable by an extension of the technique previously used (Lerche, 1985) to obtain a solution to the denumerable sum of laser modes. As we shall see later, the general solution we provide to the laser equations is expressed in terms of two arbitrary intensities $I^{+}(\nu, 0)$ and $I^{-}(\nu, 0)$ specified on $z=0$, i.e., where the incident and emergent radiation are known at one end $(z=0)$ of the laser, and the problem is to find out what is happening at the other end, say $\tau_{1}$. On the other hand a problem still of general concern is to ask what happens if both of the incident intensities $I^{+}(\nu, 0)$ and $I^{-}\left(\nu, \tau_{1}\right)$ are specified at each end of the laser; can we then determine the emergent intensitites $I^{+}\left(\nu, \tau_{1}\right)$ and $I^{-}(\nu, 0)$ ? The solution we provide later enables this problem to be linearly related to the problem we solve and so the formal answer to the question posed is affirmative. A pragmatic method of converting $I^{-}(\nu, 0)$ information to $I^{-}\left(\nu, \tau_{1}\right)$ information is, however, not that easy to achieve-although possible. We defer such calculations to elsewhere since we are currently attempting to exploit the mathematical solutions given here in astrophysical applications where such problems indeed become paramount and can be bounded by existing data on astrophysical masers.

In the case of a two-level maser, of considerable interest in astrophysical situations, Goldreich and Keeley (1972) have set up the maser equations equivalent to those given in Sec. II. In their notation,

$$
\frac{d I}{d z}=h \nu(4 \pi \Delta \nu)^{-1}\left[N_{2} A+\left(N_{2}-N_{1}\right) B I\right],
$$

where $B$ and $A$ are the Einstein coefficients and $N_{1}$ and $N_{2}$ are the number of densities of molecules in the upper and lower levels.

The rate equations are

$$
\begin{aligned}
& \dot{N}_{2}=-\left(N_{2}-N_{1}\right) B J-N_{2} A+R_{2}\left(N-N_{12}\right)-\Gamma N_{2}, \\
& \dot{N}_{1}=\left(N_{2}-N_{1}\right) B J+N_{2} A+R_{1}\left(N-N_{12}\right)-\Gamma N_{1} .
\end{aligned}
$$

Here $N=N_{1}+N_{2}, N_{12}=N_{1}-N_{2}, R_{1}$ and $R_{2}$ are the rates of pumping into levels 1 and 2 ,

$$
J=(4 \pi)^{-1} \int I d \Omega,
$$

while $d \Omega$ is the solid angle around the direction $z$ as polar axis, and $\Gamma$ is the spontaneous decay constant.

The steady-state solution of the rate equations (2) when substituted in the equation of transfer (1) gives the nondimensional form,

$$
\frac{d \mathscr{J}}{d z}=S+\beta\left(\mathscr{J}+\frac{1}{2}\right)(\beta+\mathscr{J})^{-1},
$$

where

$$
\begin{aligned}
& \mathscr{J}=B I / A, \quad \mathscr{J}=B J / A, \quad R=R_{1}+R_{2}, \quad \Delta R=R_{1}-R_{2}, \\
& \alpha=R A /(\Gamma \Delta R), \quad \beta=\frac{1}{2}(1+\Gamma / A), \quad S=\alpha \beta(1-\alpha)^{-1},
\end{aligned}
$$


and the arc length $z$ are measured in units of the unsaturated growth length, $L$, given by

$$
L=4 \pi(B h \nu)^{-1} \Delta \nu S\left(N_{1}-N_{2}\right)^{-1} .
$$

The integration over solid angle in equation (3) accounts for the effects of radiation being added to, or subtracted from, the beam direction.

Goldreich and Keeley (1972) have provided approximate solutions to equation (4) when the parametric variables $\beta$ and $S$ are (i) constant throughout a right circular cylinder of length $2 l$ and radius $\rho$, and also (ii) constant throughout a sphere of radius $R$, and elsewhere zero. The ray direction, $z$, does not have to correspond to any particular principal direction of the assigned geometry.

In the case in which the specific intensity $\mathscr{J}$ is dependent solely on one Cartesian coordinate direction (the $z$-direction) so that a one-dimensional problem results, the coefficients in equation (4) are either (a) constants (with respect to all three directions $x, y, z$ of the Cartesian coordinate system) or (b) dependent only on $z$.

In that case a direct correspondence can be made betweeen the works of Goldreich and Keeley (1972) and Cassidy (1984) as follows.

II. A continuum set of modes in one-dimension. Cassidy (1984) has pointed out that "In steady-state, and for a perfect device in which the wave guide scattering/absorption loss is zero, the $m$ th mode intensity $I_{m}^{ \pm}$in the (+) forward and (-) backward-travelling directions is found by solving the set of nonlinear coupled differential equations (Lau and Yariv, 1982; Marcuse, 1983)

$$
\begin{aligned}
\pm \frac{d I_{m}^{ \pm}}{d z}=\left\{g_{m}\left(N-n_{1} S\right) I_{m}^{ \pm}+\beta B_{m}\left[N+n_{1} \sum_{i} \sigma_{i}\left(I_{i}^{+}\right.\right.\right. & \left.\left.\left.+I_{i}\right)\right]\right\} \\
& \times\left\{S+\sum_{i} \sigma_{i}\left(I_{i}^{+}+I_{i}^{-}\right)\right\}^{-1} .
\end{aligned}
$$

The notation is that employed previously (Cassidy, 1984; Marcuse, 1983), but see note 1 for a synopsis.

To simplify later comparisons with previous work, introduce

$$
J(z)=\sum_{i} \sigma_{i}\left(I_{i}^{+}+I_{i}^{-}\right),
$$

and write equation (6) in the form

$$
\frac{d I_{m}^{ \pm}}{d z}= \pm\left\{Q_{m}+b_{m}\left(\alpha_{m}+I_{m}^{ \pm}\right)[S+J(z)]^{-1}\right\},
$$

where

$$
\begin{aligned}
& Q_{m}=\beta n_{1} B_{m}, \\
& b_{m}=g_{m}\left(N-n_{1} S\right), \\
& b_{m} \alpha_{m}=\beta B_{m}\left(N-n_{1} S\right) .
\end{aligned}
$$


To obtain the continuum representation, run the discrete index mode counter $m$ to a continuous variable so that with the equivalent replacement $I_{m}^{+} \rightarrow I^{ \pm}(\nu) d \nu$, where $I(\nu)$ is the specific intensity in the frequency band $\nu$ to $\nu+d \nu$, we can write equation (6) in the continuum form

$$
\frac{d I^{ \pm}(\nu, z)}{d z}= \pm\left\{Q(\nu)+b(\nu)\left[\alpha(\nu)+I^{ \pm}(\nu, z)\right][S+J(z)]^{-1}\right\},
$$

with

$$
J(z)=\int_{0}^{\infty} d \nu \sigma(\nu)\left[I^{+}(\nu, z)+I^{-}(\nu, z)\right],
$$

and where the rest of the notation is clear.

Equation (10) can be solved as follows.

Introduce $\tau$ as a fundamental variable replacing $z$ through

$$
d \tau=[S+J(z)]^{-1} d z,
$$

with $\tau=0$ on $z=0$.

This transformation enables us to write equation (1) in the form

$$
\frac{d I^{ \pm}(\nu, \tau)}{d \tau}= \pm\left\{\Gamma(\nu)+b(\nu) I^{ \pm}(\nu, \tau)+Q(\nu) J(\tau)\right\},
$$

where

$$
\Gamma(\nu)=b(\nu) \alpha(\nu)+S Q(\nu) .
$$

For the moment regard $J(\tau)$ as given. Then the formal solution to equation (13) is

$$
\begin{aligned}
I^{ \pm}(\nu, \tau)= & I^{ \pm}(\nu, 0) e^{ \pm b \tau}+\Gamma b^{-1}\left(e^{ \pm b \tau}-1\right) \\
& \pm Q \int_{0}^{\tau} J\left(\tau^{\prime}\right) \exp \left[ \pm b\left(\tau-\tau^{\prime}\right)\right] d \tau^{\prime},
\end{aligned}
$$

where $I^{ \pm}(\nu, 0)$ are the specific intensitites on $\tau=0$. Now add the $I^{ \pm}$equations (15), multiply the result by $\sigma(\nu)$ and integrate over all $\nu$ so that, from equations (11) and (15), we obtain a linear integral equation for $J(\tau)$ in the form

$$
J(\tau)=A(\tau)+\int_{0}^{\tau} J\left(\tau^{\prime}\right) H\left(\tau-\tau^{\prime}\right) d \tau^{\prime},
$$

where

$$
\begin{aligned}
A(\tau)=\int_{0}^{\infty} d \nu \sigma(\nu)\left[I^{+}(\nu, 0) \exp (b(\nu) \tau)+I^{-}(\nu, 0) \exp (-b(\nu) \tau)\right. & \\
& \left.+4 \Gamma(\nu) b(\nu)^{-1} \sinh ^{2}\left(\frac{1}{2} b(\nu) \tau\right)\right]
\end{aligned}
$$

and

$$
H(\tau)=2 \int_{0}^{\infty} d \nu \sigma(\nu) Q(\nu) \sinh [b(\nu) \tau]
$$


Equation (16) is of the standard convolutional type and is readily solved by Laplace transform techniques yielding

$$
J(\tau)=\mathscr{L}_{\tau}^{-1}\left[\left(\mathscr{L}_{\mu} A\right)\left(1-\mathscr{L}_{\mu} H\right)^{-1}\right],
$$

where $\mathscr{L}_{\mu}$ is the Laplace transform operator and $\mathscr{L}_{\tau}^{-1}$ its inverse.

Armed with the solution (18) for $J(\tau)$, equation (14) then yields a quadrature solution for $I^{ \pm}(\nu, \tau)$ and equation (12) in the form

$$
z=\int_{0}^{\tau} d \tau^{\prime}\left[S+J\left(\tau^{\prime}\right)\right]
$$

provides the final connection between the variables $\tau$ and $z$ converting the behavior of specific intensity to a form explicitly dependent on frequency, $\nu$, and position, $z$.

The major difference between a continuum representation and the denumerable mode behavior described previously (Cassidy, 1984) occurs in the denominator factor $1-\mathscr{L}_{\mu} H$ in equation (18).

In the continuum case we have that

$$
D \equiv 1-\mathscr{L}_{\mu} H=1-\int_{0}^{\infty} d \nu \sigma(\nu) Q(\nu)\left[(\mu-b(\nu))^{-1}-(\mu+b(\nu))^{-1}\right],
$$

whereas in the discrete mode case we obtain

$$
D=1-\sum_{i} \sigma_{i} Q_{i}\left[\left(\mu-b_{i}\right)^{-1}-\left(\mu+b_{i}\right)^{-1}\right] .
$$

The zeroes of $D$ control the response of the system since they provide pole contributions in the evaluation of $J(\tau)$.

In the discrete case, of $2 M$ modes, the denominator (20b) has $2 M$ zeroes in complex $\mu$-space independent of the specific values assigned to $\sigma_{i}, Q_{i}$ and $b_{i}$. In the continuum case, however, the number of zeroes of $D$ in complex $\mu$-space is determined by the functional forms assigned to $\sigma, Q$ and $b$ with respect to $\nu$.

This situation is precisely analogous to that occurring in plasma physics where the behavior of $2 M$ interpenetrating beams (with no net flow) leads to $2 M$ modes in the dispersion relation (the equivalent of the denominator $D=0$ ), but when the number of beams is increased so that a continuum distribution of particle velocities is allowed for, the number of modes of the dispersion relation does not increase indefinitely but rather tends to a finite limit determined by the functional shape of the velocity distribution function. A detailed exposition of this problem has been presented in Montgomery and Tidman (1968) and in Clemmow and Dougherty (1969). We argue by precise analogy here, taking over the results of such work mutatis mutandis, and see no need to repeat the details here.

This completes the solution to the one-dimensional continuum laser problem when the intrinsic functional coefficients entering equation (6), and its continuum analog, equation (10), are dependent on spatial position.

III. Discussion and conclusion. We have provided, apparently for the first time, the general solution to the steady-state one-dimensional continuum (in frequency) laser problem with spatially dependent coefficients. The general method of solution presented 
here seems not to be applicable to lasers with spatially variable coefficients in more than one dimension and, as has been shown in detail by Goldreich and Keeley (1972) in the case of astrophysical masers at least, such a spatial variation of parameters can have a profound effect on the perceived size of regions of maser activity. This general area of interest remains an outstanding concern for investigation. We suspect, but have been unable to prove, that some method of generalizing the procedure recorded here to such three-dimensional situations may be possible. This is, however, conjecture and we would be interested in seeing any further developments along such lines.

Note 1. Cassidy (1984) notes that "Equation (6) results from considering the timedependent multimode rate equations for a two-level system in which the population of the lower level $n_{1}$ is taken to be constant and the time dependence of the upper level population $n_{2}$ is described by

$$
\frac{d n_{2}}{d t}=N-\left(n_{2}-n_{1}\right) \sum_{i} \sigma_{i}\left(I_{i}^{+}+I_{i}^{-}\right)-n_{2} / \tau
$$

which may be interpreted as explaining that the time rate of change of the upper level population $n_{2}$ equals the pumping rate $N$ minus the loss of population due to stimulated and spontaneous emission events. In the equations the variables have the following definitions: $\tau$ is the spontaneous transitions lifetime of the upper state, $B_{m}$ is the spontaneous emission profile and specifies which fraction of the spontaneous events falls within the wavelength interval of the $m$ th mode, and $\sigma_{i}$ is a saturation profile which denotes the efficiency of the $i$ th mode intensity in reducing the inversion. Equation (6) results from setting the time derivative $d n_{2} / d t$ equal to zero, solving for the steady-state population $n_{2}$ and letting the gain be proportional to the population inversion $\left(n_{2}-n_{1}\right)$ and the spontaneous emission proportional to the upper level population $n_{2}$. Equation (6) allows for the possibility that the $m$ th mode gain coefficient, $g_{m}$, may not equal $\sigma_{m}$, sets $\tau^{-1}=S$, and allows for the fact that only a fraction $\beta$ of the spontaneous emission events couples into the laser mode."

\section{REFERENCES}

[1] D. T. Cassidy, Appl. Phys. Lett. 44, 489, 1984

[2] P. C. Clemmow and J. A. Dougherty, Plasma Electrodynamics, McGraw-Hill Book Co., Reading, Mass., 1969

[3] A. Cook, Celestial masers, Cambridge University Press, Cambridge, 1979

[4] P. Goldreich and S. Keeley, Astrophys. J. 174, 517, 1972

[5] K. Y. Lau and A. Yariv, Appl. Phys. Lett. 40, 763, 1982

[6] I. Lerche, J. Math. Phys., 26, 1858, 1985

[7] D. Marcuse, J. Quantum Electron QE-19, 63, 1983

[8] D. C. Montgomery and D. A. Tidman, Plasma kinetic theory, McGraw-Hill Book Co., Reading, Mass., 1968 